\title{
Vedolizumab bei Morbus Crohn (MC) und Colitis ulcerosa (CU)
}

Interview mit Univ.-Prof. Dr. Shomron Ben-Horin vom Sheba Medical Center in Israel anlässlich einer

Roadshow durch Österreich im Frühjahr 2018.

Darmselektive vs. systemische CEDBehandlung

Ben-Horin: Bezüglich Effektivität und Sicherheit gibt es wahrscheinlich einen biologischen Grund dafür, dass eine darmselektive Behandlung einen zusätzlichen Nutzen zu vermitteln scheint. Aufgrund des Mangels von Head-to-Head-Studien ist es allerdings schwierig, das endgültig zu bestätigen.

Was zeigen aktuelle Real-World-Daten vs. RCT-Daten für Vedolizumab bei $M C$ ?

Ben-Horin: In gewisser Weise spiegeln die RWE-Daten die bereits vorhandenen RCT-Daten bezüglich der Effektivität zu Vedolizumab wider. In der Behandlung von Biologikanaiven Patienten - einer Subgruppe des GEMINI-Programms - sehen wir sogar geringfügig bessere RWEErgebnisse unter Vedolizumab als Erstlinien-Biologikatherapie. Und im Gegensatz zu den RCT-Daten haben die RWE-Daten eine ähnliche, sehr gute Effektivität sowohl für MC- als auch CU-Patienten gezeigt. Vermutungen aus dem GEMINI-Programm, dass bei MC-Patienten eine verminderte Effektivität zu befürchten wäre, wurden also widerlegt das scheint in der klinischen Praxis nicht der Fall zu sein.

\section{Vedolizumab und Mukosaheilung}

Ben-Horin: Die Evidenz, die wir bislang aus RCT- und den jüngsten RWE-Daten haben, scheint recht vergleichbar damit, was unter anti-TNF-Wirkstoffen zu beobachten war. In manchen Fällen, z. B. in der US-amerikanischen Victory-Kohorte, schien es Hinweise auf eine ver- zögerte Mukosaheilung zu geben, die maximale Mukosaheilung wurde erst nach 22-wöchiger Behandlung erreicht. Wir müssen uns hier wahrscheinlich in Geduld üben und benötigen außerdem diesbezüglich noch mehr qualitativ hochwertige, kontrollierte Daten.

Was haben Patienten bezüglich der Effektivität von Vedolizumab zu erwarten?

Ben-Horin: Auf Basis der GEMINIAnalyse wissen wir, dass vor allem bei MC-Patienten eine Behandlungsdauer von 10 bis 14 Wochen erforderlich sein kann, bevor man eine Wirkung sieht - das beobachten wir übrigens auch in der klinischen Praxis. So lange sollte man also mindestens warten, bevor man entscheidet, ob man die Behandlung weiterführt oder abbricht. Wir führen in manchen Fällen auch eine Bridging-Therapie mit Budesonid oder niedrigdosierten Kortikosteroiden durch und entscheiden dann erst nach rund 22 Wochen. Eine gewisse Geduld ist also vonnöten, genauso wie bei anderen Biologika. In meiner klinischen Praxis habe ich allerdings auch Biologika-naive Patienten, die bereits nach der zweiten Infusion eine wesentliche Verbesserung angaben - und das waren Patienten mit blutiger Diarrhö, wo man also einen Placeboeffekt eher ausschließen würde.

Talspiegel messen bei Vedolizumab? Ben-Horin: Peaking-Probleme und Probleme mit der Immunogenität sind bei Vedolizumab sehr viel seltener als unter anti-TNF-Wirkstoffen.
Welche MC-Patienten haben den größten Nutzen einer Behandlung?

Ben-Horin: Ältere Patienten oder solche mit systemischen Problemen wie Infekten oder junge Männer unter TNF-Behandlung sind diejenigen Patienten, bei denen ich aufgrund der Sicherheit eher geneigt wäre, Vedolizumab einzusetzen.

Schlüsselergebnisse der kürzlich von Ihnen veröffentlichten pan-europäischen RWE-Studie

Ben-Horin: Bei Biologika-naiven Patienten, die also Vedolizumab als Erstlinien-Biologikum erhielten, lag die Gesamtansprechrate bei Induktion bei fast $80 \%$, die Remission im Bereich von $45 \%$ und die kortikosteroidfreie Remission bei rund $35 \%$. Das sind wirklich beeindruckende Daten. Einige meiner besten Erfolge sind allerdings auch Patienten, die von einem anderen Biologikum umgestiegen sind.

Der Nutzen von Vedolizumab in einem Satz?

Ben-Horin: Vedolizumab hat insgesamt eine sehr ähnliche Effektivität wie die Wirkstoffe der anti-TNF Klasse, die Sicherheit ist allerdings besser.

\section{Weiterführende Literatur}

Feagan et al (2013) NEJM 396(8):699-710 Sandborn et al (2013) NEJM 396(8):711-721 Kopylov et al (2016) IBD 23:404-408

J. Gastroenterol. Hepatol. Erkr. 2018 · 16:70 https://doi.org/10.1007/s41971018-0030-1

(c) Springer-Verlag GmbH Austria, ein Teil von Springer Nature 2018 